\title{
Chronic Neurobrucellosis Investigated via Metagenomic Next-generation Sequencing
}

\author{
Yun Zhang, Xiaodan Shi, Xiaofei Fei, Guodong Feng, Gang Zhao* \\ Department of Neurology, Xijing Hospital, Fourth Military Medical University, Xi'an, China \\ E-mail: zhaogang@fmmu.edu.cn
}

\begin{abstract}
We found that NGS technology has great significance for the diagnosis of brucellosis in non-epidemic area and can rule out other infections. The number of Brucella gene fragments detected by NGS may be affected by clinical resistance to Brucella treatment and Brucella infection. The sensitivity of SAT to detect Brucella may is limited.

Keywords: central nervous system infection, cerebrospinal fluid, cetagenomic second-generation sequencing technology, diagnosis
\end{abstract}

\section{Introduction}

Brucellosis is a zoonotic disease, with goats the main source of infection ${ }^{[1]}$. Brucella infection often involves multiple organ systems, with neurobrucellosis a rare complication that has diverse clinical manifestations ${ }^{[2]}$. At present, the methods for detection of Brucella are; serum agglutination test, polymerase chain reaction (PCR), and enzyme-linked immunosorbent assay (ELISA) ${ }^{[3]}$. Although culture isolation of Brucella is the gold standard, positive rates are very low. Further, neurological brucellosis is often confused with tuberculosis, cryptococcosis, and peripheral neuropathy, resulting in common misdiagnosis ${ }^{[4]}$.

Next Generation Sequencing (NGS) technology is a new method for pathogen detection within the central nervous system $^{[5,6,7]}$. Herein, We studied the significance of NGS in the diagnosis of brucellosis in non-epidemic areas.

\section{Materials and methods}

DNA extraction and sequencing:

200- $\mu$ l of cerebral spinal fluid (CSF) was collected from four patients and negative controls in new sterile tubes, frozen immediately, and DNA purified with TIANGEN DP316. DNA density and quality were assessed by Qubit and agarose gel electrophoresis. A sequencing library was prepared using a NEB Next Ultra II DNA library Prep Kit for Illumina according to the manufacturer's instructions.

\section{Results}

Clinical Findings:

Four patients were 3 male and 1 female, aged between 27 and 53 years. The clinical features are summarized in Table 1 .

Table 1. Clinical features of the four patients with neurobrucellosis

\begin{tabular}{cccccccccccc}
\hline $\begin{array}{c}\text { Case } \\
\text { numbe }\end{array}$ & Age & Sex & Fever Sweating & $\begin{array}{c}\text { Hearing } \\
\text { loss }\end{array}$ & $\begin{array}{c}\text { Joint } \\
\text { pain }\end{array}$ & $\begin{array}{c}\text { Meningeal } \\
\text { signs }\end{array}$ & Tinnitus & $\begin{array}{c}\text { Lower } \\
\text { limb } \\
\text { weakness }\end{array}$ & $\begin{array}{c}\text { Unsteady } \\
\text { walking }\end{array}$ & Diplopia \\
\hline 1 & 30 & Male & + & - & + & - & - & + & + & + & - \\
2 & 52 & Male & - & - & - & + & - & - & + & + & - \\
3 & 27 & Female & + & - & - & - & - & - & + & - & + \\
4 & 43 & Male & - & + & + & + & - & + & + & - & - \\
\hline
\end{tabular}

+ positive; - negative 


\subsection{Case 1}

On the first day, physical examination identified; bilateral hearing damage, level 5 lower limb muscle strength, and an active lower limb tendon reflex, with no other obvious abnormality. On the 34th day, cerebrospinal fluid cytology showed the white blood cell count to be $284 / \mathrm{mm} 3$, neutrophils $36 \%$, lymphocytes $61.5 \%$, and monocytes $2 \%$. Biochemistry: sugar $2.97 \mathrm{mmol} / \mathrm{L}$ and protein $2.51 \mathrm{~g} / \mathrm{L}$. Cerebrospinal fluid acid-fast staining, T-spot, X-spot, and cerebrospinal fluid M. tuberculosis DNA test were negative. Brucella standard tube agglutination was negative. At this point, the diagnosis of tuberculosis was not established, and NGS was requested. On the 38th day, sequencing results were reported. A Brucella gene fragment was identified and the patient treated with empirical anti-Brucella therapy. The cerebrospinal fluid Brucella agglutination test was repeated at the same time.

\subsection{Case 2}

The patient was male, 52 years of age and was admitted to the Department of Neurology of Xijing Hospital on December 8, 2017 for "four months of weakness, numbness, and pain in both lower limbs".

Physical examination: bilateral bicep reflex, tricep reflex, and knee reflex were active, with bilateral Babinski sign positive. Antiviral and hormone therapy were administered. On the 8th day, the patient's symptoms were relieved. Magnetic resonance imaging (MRI) of the head revealed changes in the fifth and sixth ventricles, the bilateral anterior and posterior ventricles, the anterior and posterior horns, and demyelination of the right frontal lobe. These results combined with the medical history did not rule out multiple system atrophy, hence NGS was requested for Brucella. On the $10^{\text {th }}$ day, after lumbar puncture the Brucella standard tube agglutination was positive. The patient was told to adhere to the doctor's Brucella treatment regimen with the Brucella agglutination test to be repeated after 6 months.

\subsection{Case 3}

The patient was admitted to the Department of Neurology with "peripheral neuropathy". Physical examination: double upper limb muscle strength of 5, double lower limb muscle strength of 4, normal muscle tension, active tricep reflex. All the examinations is negative. There was a large cyst in the occipital region, but the MRI scan of the brain and fluid-attenuated inversion recovery (FLAIR) identified no abnormalities. On the eighth day, with continued similar treatment, there was no improvement in the patient's condition, so NGS of cerebrospinal fluid was requested. On the 10th day, NGS identified Brucella. Blood and cerebrospinal fluid were sent for Brucella culture and agglutination test with the cerebrospinal fluid found to be positive by the Brucella standard tube agglutination test. The patient was treated for Brucella and informed to adhere to medical treatment outside the hospital.

\subsection{Case 4}

Physical examination: hearing loss in both ears, grade 3 muscle strength in both lower limbs. Sensory decline below the bilateral groin, the lower limbs reflected active, bilateral Babinski signs were positive, and meningeal irritation was negative. General examination after admission was normal. On the 3rd day, the symptoms did not improve, and urination at night was uncontrolled. Thoracic spine MRI: abnormal signal in the posterior thoracic spinal cord. In order to exclude patients from coinfection, we sent the patient's cerebrospinal fluid for second-generation sequencing. On the 6th day, NGS identified Brucella, although the standard tube agglutination test was negative. Medical history identified cattle and sheep contact with a diagnosis of neurobrucellosis spinal cord type.

\section{NGS results}

DNA fragments of Brucella were found by NGS in the cerebrospinal fluid of four patients. The specific gene fragments of the sequenced Brucella were between 4 and 90, with coverage ranging from 0.0977 to 0.5762. NGS detailed information is summarized in Figure 2. 

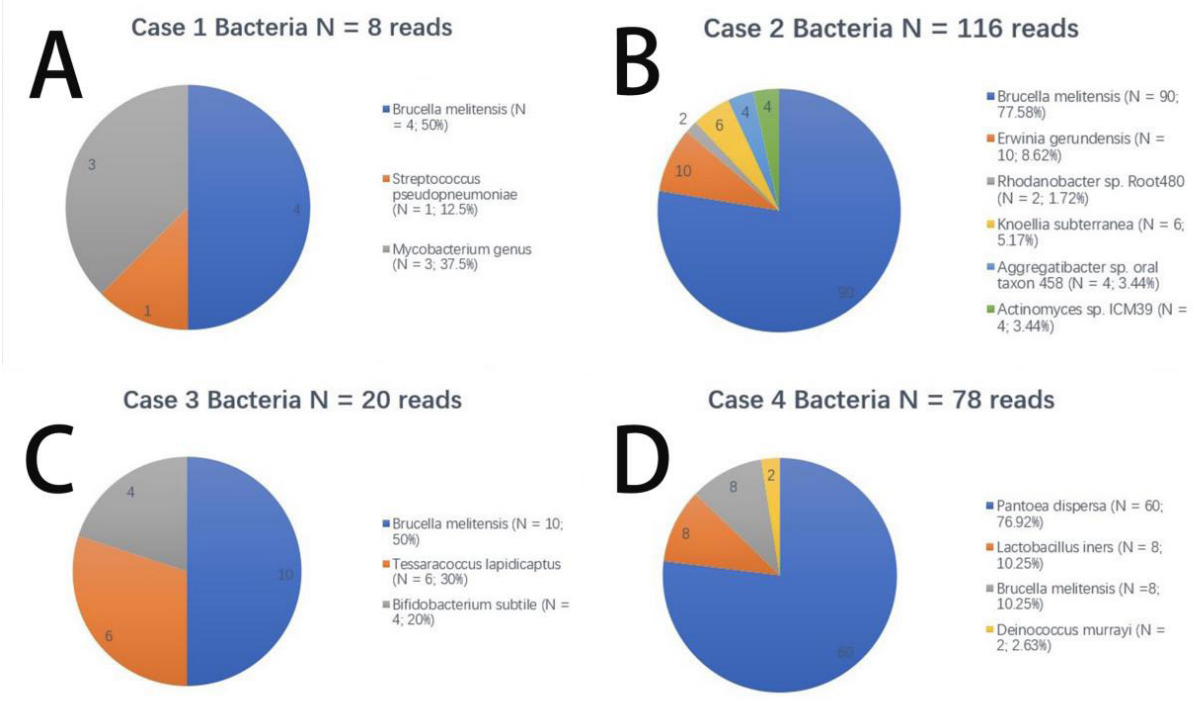

Figure 2. Next-generation sequencing (NGS) of cerebrospinal fluid (CSF) in the four patients. The number of sequences reads identified corresponding to Brucella species was (A) 4 (case 1), (B) 90 (case 2), (C) 10 (case 3), and (D) 60(case 4)

\section{Discussion}

Three of the four patients lived in the region non-epidemic with brucellosis and one lived in the region epidemic with brucellosis. Because of the large population in the non-epidemic areas, the China government has not carried out the routine screening of Brucella because of the economic costs. The clinical manifestations of the three non- epidemic area patients were similar to those of other neurological infectious diseases, hence three of the patients were not initially considered to have brucellosis infections, but rather were assessed for multiple sclerosis and tuberculosis, viral meningoencephalitis, or peripheral neuropathy ${ }^{[8,9]}$. After the second-generation sequencing, we reviewed the cerebrospinal fluid SAT test and found it to be positive. It shows that NGS can play a huge role in the diagnosis of Brucella in non- epidemic area patients ${ }^{[7]}$. The fourth case is a case of refractory neuropathic brucellosis in the epidemic area. The main reason is to exclude the infection of other bacteria or DNA virus ${ }^{[10]}$. The infection is still considered to be Brucella infection, and the evidence of other coinfections is insufficient.

In addition to the third case, the other three cases of Brucella fragments were less in the sequencing results. There were two reasons why the number of Brucella fragments detected by NGS in 3 cases was less: First, it is related to the drug which patient used before sequencing. Second, the meningeal infection is relatively mild. Most importantly, there are three considerations regarding the problems of other bacteria in the sequencing results: First, the pollution during the experiment, which is also a huge challenge for all sequencing problems, in this study, a negative single-shot experiment was performed at the same time as sequencing to eliminate the effects of pollution. Second, perhaps the symbiotic bacteria of the human body . Third, the composite infection, the detected bacteria may be combined with Brucella to cause a composite infection.

\section{References}

[1] Ariza J, Bosilkovski M, Cascio A, Colmenero JD, Corbel MJ, Falagas ME, et al. Perspectives for the treatment of brucellosis in the 21st century: the Ioannina recommendations. PLoS Med. 2007; 4: e317.

[2] Rossetti CA, Arenas-Gamboa AM, Maurizio E. Caprine brucellosis: A historically neglected disease with significant impact on public health. PLoS Negl Trop Dis. 2017; 11: e5692.

[3] Mohseni K, Mirnejad R, Piranfar V, Mirkalantari S. A Comparative Evaluation of ELISA, PCR, and Serum Agglutination Tests For Diagnosis of Brucella Using Human Serum. Iran J Pathol. 2017; 12: 71-76.

[4] Zheng R, Xie S, Lu X, Sun L, Zhou Y, Zhang Y, Wang K. A Systematic Review and Meta-Analysis of Epidemiology and Clinical Manifestations of Human Brucellosis in China. Biomed Res Int. 2018.

[5] Wilson MR, Naccache SN, Samayoa E, Biagtan M, Bashir H, Yu G, et al. Actionable diagnosis of neuroleptospirosis by next-generation sequencing. $N$ Engl J Med. 2014; 370: 2408-17.

[6] Mongkolrattanothai K, Naccache SN, Bender JM, Samayoa E, Pham E, Yu G, et al. Neurobrucellosis: Unexpected Answer From Metagenomic Next-Generation Sequencing. J Pediatric Infect Dis Soc. 2017; 6: 393-398. 
[7] Fan S, Ren H, Wei Y, Mao C, Ma Z, Zhang L, et al. Next-generation sequencing of the cerebrospinal fluid in the diagnosis of neurobrucellosis. Int J Infect Dis. 2018; 67: 20-24.

[8] Dasari S, Naha K, Prabhu M. Brucellosis and tuberculosis: clinical overlap and pitfalls. Asian Pac J Trop Med. 2013; 6: $823-825$

[9] Ulu-Kilic A, Metan G, Alp E. Clinical presentations and diagnosis of brucellosis. Recent Pat Antiinfect Drug Discov. 2013; $8: 34-41$.

[10] Tang Y, Lin L, Sebastian A, Lu H. Detection and characterization of two co-infection variant strains of avian orthoreovirus (ARV) in young layer chickens using next-generation sequencing (NGS). Sci Rep. 2016; 6: 24519. 